\title{
Experimental Study on droplet generation in flow focusing devices considering a stratified flow with viscosity contrast
}

\author{
Xiaoming Chen and Carolyn L. Ren* \\ Department of Mechanical and Mechatronics Engineering, University of Waterloo, 200 University Ave \\ W., Waterloo, ON, N2L 3G1, Canada \\ * Corresponding author. \\ Tel.: 1-519-888-4567 x 33030; \\ fax: 1-519-885-5862; \\ e-mail: c3ren@uwaterloo.ca

\section{Highlight} \\ - Droplet generation dynamics is experimentally studied in a simple flow focusing generator with two junctions in series by systematically considering \\ five scenarios where the viscosity contrast between two miscible fluids which form the dispersed phase of droplets is varied. \\ - Droplet sizes are characterized for the five scenarios and physical models for droplet size are obtained for each scenario based on scaling laws. \\ - The results indicate that different scenarios have different impact on droplet generation and thus find different applications. \\ - The scenario with high viscous fluid surrounded by low viscous fluid allows particles to be focused into a thin stream line before droplet generation, \\ which is helpful for improve single particle encapsulation. \\ - The scenario with two fluids side by side in the first junction generates droplets with high monodispersity in the largest range of flow ratios.
}

\begin{abstract}
High throughput analysis is highly demanded in a variety of chemical reactions. Droplet microfluidics offers unique advantages over traditional multi-well plate systems for high throughput analysis such as providing a confined and more controllable environment for single particle or cell analysis. Driven by the need to improve the efficiency of encapsulating one particle or cell into one individual droplet without complicating geometric and operating conditions, this study experimentally investigated the effects of viscosity contrast between two miscible fluids that together serve the dispersed fluid on the ordering of particles before they are encapsulated into droplets by another immiscible fluid. Five scenarios with different viscosity contrast were systematically considered and a physical model of droplet size for each scenario was developed based on experimental results and scaling laws. The five different scenarios include two with pure $10 \%$ glycerol and pure $80 \%$ glycerol as the dispersed phase, respectively, and three others where these two fluids are either side by side or one is accompanied by the other. Droplet size and formation period for these scenarios were compared and analyzed considering the same geometric and flow conditions. It is found that the stratified flow structures formed in the first junction by the two miscible fluids (10\% and $80 \%$ glycerol solutions) strongly influence droplet formation dynamics such as droplet size and formation frequency. Each scenario finds its own applications. The scenario with $80 \%$ glycerol surrounded by $10 \%$ glycerol provides the optimized means for particle encapsulation. However, the scenario with two fluids side by side in the first junction generates droplets with high monodispersity for the largest range of flow ratios, which is useful for high throughput reactions involving different reagents.
\end{abstract}

\section{Introduction}

Droplet-based microfluidics has drawn ever-increasing attention due to its capability to perform high-throughput combinatorial testing for biological, chemical, and material applications (Haeberle \& Zengerle 2007; Casadevall i Solvas \& DeMello 2011; Lagus \& Edd 2013; Rosenfeld et al. 2014), such as polymerase chain reactions (PCR) (Tewhey et al. 2009; Schaerli et al. 2009), cell culture (Kwapiszewska et al. 2014), drug screening (Miller et al. 2012), and synthesis of microbeads (Shepherd et al. 2006) and nanoparticles (Shestopalov et al. 2004; Wacker et al. 2012; Kim et al. 2013). Droplet formation is the first step of all the applications and have significant impact on other associated functions such as single particle encapsulation which makes droplet microfluidic platforms more advantageous than traditional well-plate systems for offering confined and well controlled microenvironment and following processes such as merging, splitting and sorting of droplets which are required in many practical applications. Single (bio)particle (considering both polymeric or glass beads and biological cells) encapsulation is often involved in the droplet 
formation step (Köster et al. 2008; Edd et al. 2008; Velasco et al. 2012; Wu et al. 2013; Kemna et al. 2012; Abate et al. 2009; Rossow et al. 2012), where (bio)particles are suspended in the dispersed fluid and later encapsulated in droplets when the dispersed fluid interacts with the immiscible continuous fluid. Droplet formation dynamics influences the resulted encapsulation rate and performance significantly. Another example of the importance of droplet generator performance is its impact on droplet sorting downstream. It was found that slight variation in droplet spacing could cause significant disturbance on droplet sorting pattern downstream (Glawdel et al. 2011) even droplets are generated uniformly because the high speed of droplet formation and transport leaves almost no room for uncertainties. Therefore, to facilitate the progress adopting droplet microfluidics as highthroughput screening analysis tools, several challenges should be addressed coherently for droplet generators which include achieving low polydispersity of droplet size, high robustness, simplicity of the design and operation and ideally high single particle encapsulation rate.

Benefiting from the confinement that microchannel network offers, monodispersed droplets can be generated (i.e. $<3 \%$ in volume) with well controlled surface wettability and within the squeezing to transition regimes. Jetting regime raises the challenges of generating monodispersed droplets due to its high speed of generation and being prone to the classic RayleighPlateau instability (Fu et al. 2012). Simple geometries such as Tjunctions in series and flow focusing junctions in series have been employed and widely studied for droplet generation and manipulation. This study focuses on the flow focusing junctions in series because it offers symmetric control over the flow at junctions resulting in higher robustness and thus being more practically useful. Single (bio)particle encapsulation remains challenging and drives the exploration of the optimization of geometric and operating conditions for droplet generation, which could offer improved single encapsulation rate without increasing the design complexity and compromising robustness.

In general, when particles are randomly distributed in the dispersed phase, the number of particles encapsulated in droplets follows the Poisson distribution (Köster et al. 2008). Therefore, the particle concentration is often highly diluted to ensure singleparticle encapsulation, which however results in a large number of empty droplets and thus increases the waste of reagents, and the need for additional droplet sorting procedures (Velasco et al. 2012; $\mathrm{Wu}$ et al. 2013). To increase the rate of single particle encapsulation, a number of approaches have been reported. Edd et al. (Edd et al. 2008) used hydrodynamic self-organization within a high aspect-ratio microchannel to distribute evenly spaced cells, and then synchronize cell encapsulation with the frequency of droplet generation, resulting in a rate of single cell encapsulation as high as $80 \%$. Another work followed the similar cell selforganization mechanism by using Dean flow in a curved microchannel and achieved an efficiency of single cell encapsulation up to $77 \%$ (Kemna et al. 2012). Although these methods are excellent in achieving high efficiency for single cell encapsulation, the use of high flow rates generally required for self-ordering encapsulation makes the system sensitive to inherent and induced uncertainties because the system operates in dripping to jetting transitions.

Alternatively, the strategy of modifying the dispersed phase or the (bio)particles using gel-based solutions has been used for encapsulation. For example, a close-packed ordering method was reported by Abate et al. (Abate et al. 2009), which encapsulated compliant gel particles into individual droplets using a configuration with two flow-focusing junctions in series and achieved an efficiency of encapsulation as high as $98 \%$. This method could be employed for encapsulation of cells or particles into droplets; However, cells or particles must be encapsulated into gels first. Polymer hydrogels have also been utilized to encapsulate cells in a similar configuration (Rossow et al. 2012), where the mixture of hydrogel solutions and cell suspension is the dispersed phase while paraffin oil is the continuous phase. This configuration allows the chemical composite and concentration of the dispersed phase to be well controlled in the first junction and eventually in the droplet generated in the second junction. In addition, the dispersed phase is accompanied by the continuous phase on both sides resulting in more symmetric shearing which improves stability and control over droplet formation. Many existing studies on droplet generation in flow focusing geometries considered uniform viscosity between different flow streams that form the dispersed phase (Cubaud \& Mason 2008; Lee et al. 2009; Garstecki et al. 2006; Fu et al. 2009; van Steijn et al. 2010; Glawdel et al. 2012; Glawdel et al. 2012; Chen et al. 2015; Lu et al. 2014; Bai et al. 2016) with few considering the influence of viscosity contrast between different flow streams on droplet formation and control as encountered in the practical applications for cell encapsulation. Viscosity contrast between the dispersed fluids would influence droplet formation dynamics and potentially offer unique strategies for improving particle ordering before they are encapsulated into droplets and thus improve the efficiency for single encapsulation.

To this end, systematically investigating the effects of viscosity contrast between two miscible fluids which together form the dispersed fluid on droplet formation in a flow focusing geometry and evaluating its impact on single encapsulation is very important (Cubaud et al. 2012), which prompts the study presented here. It is expected that the output of this study would also provide operational guidance to improve their robustness for practical applications. In this study, we consider a configuration with two flow-focusing junctions in series where water-cosolvent mixtures, which have been widely used for pharmaceutical and cosmetic applications, are the dispersed phase and a low-viscosity silicone oil is the continuous phase. Among the commonly used cosolvents (Jouyban 2008) such as ethanol, propylene glycol, glycofural and polyethylene glycols, and glycerol, glycerol is chosen in this study because the viscosity of glycerol and water mixtures could be varied significantly by changing the concentration of glycerol while their interfacial tension remains roughly the same. In this work, we experimentally investigate droplet formation by varying the concentration of glycerol and water (by weight) which results in as large as 32 in viscosity contrast between flow streams that form the dispersed phase while maintaining a similar interfacial tension between the dispersed and continuous phase. The measured values are provided later. Droplet generation process was captured by using a high-speed camera, and droplet volumes were measured to study the droplet generation dynamics by varying the flow rate ratio, the capillary number and inlets of the dispersed phases.

\section{Materials and method}

For all experiments, a low-viscosity silicone oil (DC200, Sigma Aldrich, 10 cst) was used as the continuous phase. Two glycerol/water mixtures ( $10 \%$ and $80 \%$ in wt $\%$ ) were used to form the dispersed phases with different scenarios as illustrated in Figure 1. Viscosity was measured at room temperature $\left(22^{\circ} \mathrm{C}\right)$ using a programmable rheometer (LVDV-III Ultra CPE, Brookfield Instruments) with a cone-plate spindle (CPE-40). Interfacial tension was measured using a Wilhemy plate tensiometer (Data Physics, DCAT 11). No surfactant was added to either of the phases to avoid the influence of mass transfer effects 
on the interfacial tension. The measured viscosity and interfacial tension for the $10 \%$ glycerol/water mixture $(10 \% \mathrm{Glyc})$ were 1.37 $\mathrm{mPa} . \mathrm{s}$ and $37.1 \mathrm{mN} / \mathrm{m}$, respectively and for the $80 \%$ glycerol $/$ water mixture $(80 \% \mathrm{Glyc})$ were $44.1 \mathrm{mPa} . \mathrm{s}$ and $34.8 \mathrm{mN} / \mathrm{m}$, respectively. The microchannels were fabricated using polydimethylsiloxane (PDMS) via standard soft-lithography techniques. penetrate into the second junction. In the design, the distance was set to be $300 \mu \mathrm{m}$ after preliminary testing. The cross-sectional shape of the microchannel is rectangular and the channel depth for each chip is assumed to be uniform. Since silicone oil swells PDMS, the actual dimensions of the microchannels are smaller than the nominal dimensions and therefore were experimentally

(a)

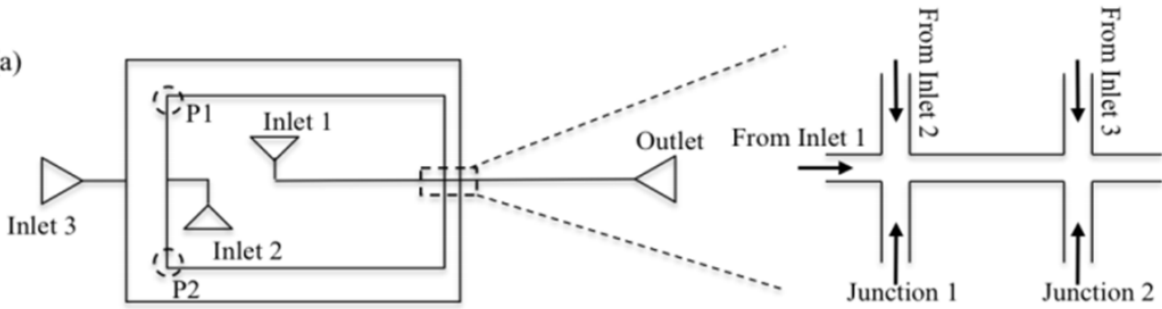

(b)

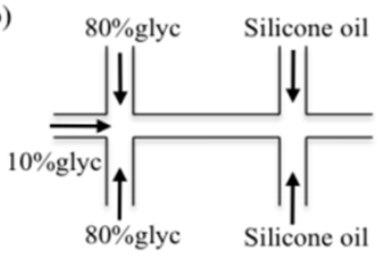

Case I

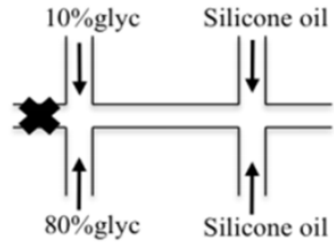

Case II
Junction 1

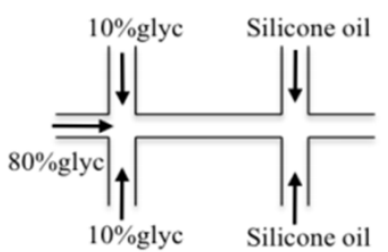

Case III

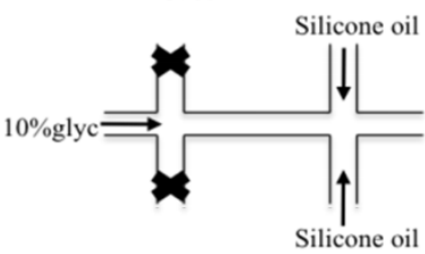

Ref Case I

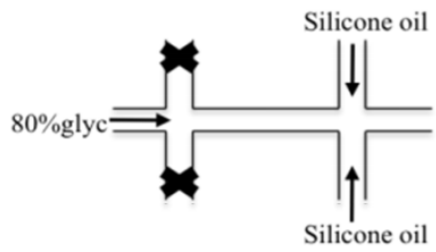

Ref Case II

Figure 1. (a) Sketch of the microchannel design. The channel width is set to be $100 \mu \mathrm{m}$, the height $40 \mu \mathrm{m}$ and the distance between the two junctions $300 \mu \mathrm{m}$. Inlets 1 and 2 are for the dispersed phase, and inlet 3 is for the continuous phase. (b) Sketch of flow combinations at the flow focusing junctions

The flow system consists of a high precision microfluidic pressure control system (MSFC 8C, Fluigent) and three in-line flow sensors (SLG 1430, Sensirion). These flow sensors are able to measure water flow rates up to $40 \mu \mathrm{L} / \mathrm{min}$ with a frequency of $100 \mathrm{~Hz}$, and silicone oil and glycerol/water mixtures up to $5 \mu \mathrm{l} / \mathrm{min}$. The calibration process can be found elsewhere (Chen et al. 2015). We used the pressure control system and the flow sensors because syringe pumps cause unsteadiness in flow rates due to ratcheting of the stepper motor and imperfections in the drive screw (Korczyk et al. 2011). Droplet formation was visualized using an inverted epifluorescence microscope (Eclipse Ti, Nikon) with a 20x objective and a high speed camera (Phantom v210, Vision Research). The frame rate for each video was set to be $5000 \mathrm{fps}$. The detailed experimental setup can be found elsewhere (Glawdel et al. 2012). For each scenario with a particular viscosity contrast, different input pressures were applied to span a large range of capillary number and flow rate ratios. The flow rates of each experimental scenario are provided in the supplemental material.

The channel network as shown in Figure 1(a) is designed to ensure that its performance is insensitive to uncertainties such as flow rate fluctuations and tubing compliances and the required pressure is within the limit of the pressure system which is lbar. In brief, the distance between the two junctions cannot be too long so that the diffusion between the dispersed phases can be neglected. On the other hand, the distance should be long enough to make sure that the stratified flow is fully developed before fluids measured after the PDMS channels were sufficiently swollen. The actual channel width and height were measured as $88 \mu \mathrm{m}$ and 36.3 $\mu \mathrm{m}$, respectively. The method for measuring channel height can be found elsewhere (Glawdel et al. 2012).

The flow scenarios are illustrated in Figure 1(b). During the droplet formation process, the glycerol mixtures were injected into the first flow-focusing junction from inlet 1 and 2 which formed a stratified flow at the first junction. The stratified flow stream was then squeezed into droplets by the continuous phase in the second junction. There are three combinations of the stratified flow structure corresponding to the two miscible liquids flowing into the first junction. In Case I, the low viscosity liquid ( $10 \% \mathrm{glyc})$ is pumped into the first junction from inlet 1 , and the high viscosity liquid $(80 \% \mathrm{glyc})$ is pumped into the first junction from inlet 2 , which form a sandwiched structure with the $10 \%$ glyc in the middle and $80 \%$ glyc accompanying its two sides. In Case II, both inlet 1 and 2 are blocked and two new inlets are made at P1 and P2 through which $10 \%$ glyc and $80 \%$ glyc are pumped respectively forming a stratified flow side by side. In Case III, $80 \%$ glyc is pumped from inlet 1 and $10 \%$ glyc from inlet 2 forming a sandwiched structure, opposite to Case I with $80 \%$ glyc surrounded by $10 \%$ glyc. In Ref Case I and II, inlet 2 is blocked while pure $10 \%$ glyc and $80 \%$ glyc are pumped into the first junction, respectively. Each structure finds its own applications. For example, the sandwiched structure allows (bio)particles to be focused and spaced apart so that encapsulation is controllable while the stratified flow structure is useful for reactions involving different reagents. 


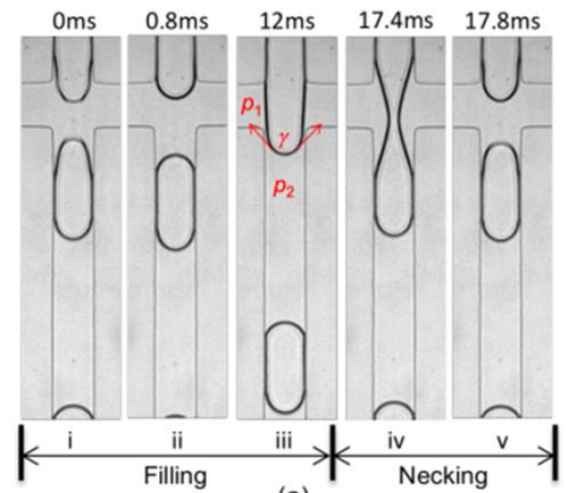

(a)

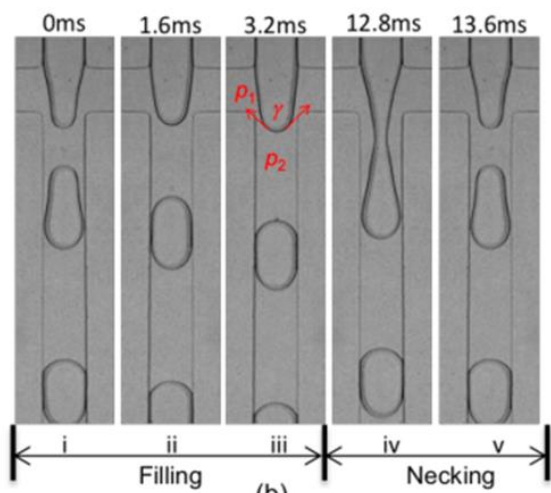

(b)

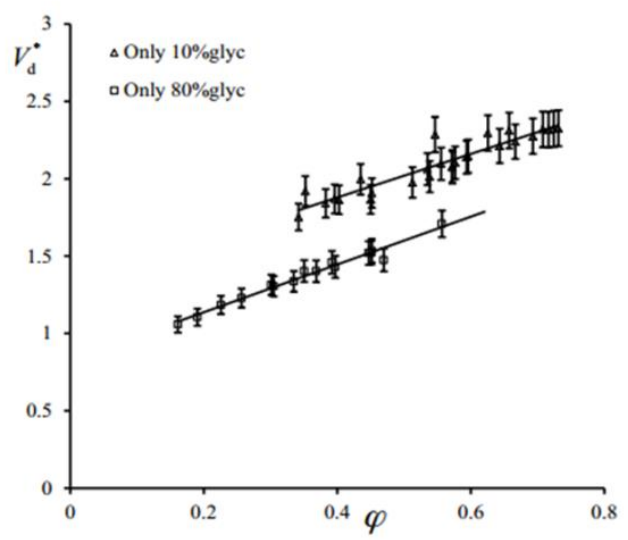

Figure 2. Droplet generation process (i) Droplet pinches off; (ii) The dispersed phase returns back to the semilunar shape due to interfacial tension and starts to fill the main channel; (iii) End of filling stage where the silicone oil starts to squeeze the dispersed phase; (iv) The necking width reaches a critical value, and the droplet starts to pinch off; (v) The current droplet pinches off, and a new droplet is forming. (a) Pure $10 \%$ glyc as the dispersed phase with the flow rate of silicone oil and $10 \% \mathrm{glyc}$ as $Q_{\text {oil }}=4 \mu \mathrm{l} / \mathrm{min}$ and $Q_{10 \% g l y c}=1.62 \mu \mathrm{l} / \mathrm{min}$, respectively; (b) pure $80 \% \mathrm{glyc}$ as the dispersed phase with the flow rate of silicone oil and $80 \% \mathrm{glyc}$ as $Q_{\text {oil }}=4 \mu \mathrm{l} / \mathrm{min}$ and $Q_{80} \% \mathrm{glyc}=1.62 \mu \mathrm{l} / \mathrm{min}$, respectively; and (c) The relationship between the measured droplet volume scaled as $V_{d}^{*}=V_{d} / w^{2} h$ and flow rate ratio $\varphi=Q_{d} / Q_{c}$, where the linear trend line for the cases of pure $10 \%$ glyc and $80 \%$ glyc are predicted by $V_{d}^{*}=1.31+1.41 \varphi$ and $V_{d}^{*}=0.83+1.55 \varphi$, respectively.

In order to fully understand the influence of viscosity contrast of two dispersed fluids on droplet generation, experimental results obtained for The Case I - III are compared with that obtained from the cases without stratified flow structures - Ref Case I and II, respectively.

\section{Experimental results and discussions}

\subsection{Droplet generation with either pure $10 \%$ glyc or pure $80 \%$ glyc as the dispersed phase}

To compare the droplet generation process between two scenarios - using a stratified flow with a viscosity contrast as the dispersed phase and using pure $10 \%$ glyc or $80 \%$ glyc solution as the dispersed phase, experimental conditions such as channel layout and geometry and flow rates were kept the same. Figure 2(a) and 2(b) show an example of droplet formation cycle which consists of three stages for both Ref Case I and II, respectively. First, a filling stage $(\mathrm{i} \rightarrow \mathrm{iii})$, where the dispersed phase returns to a semilunar shape due to the interfacial tension after the dispersed phase pinches off and starts filling the main channel. The interface continues to grow while the continuous phase bypasses the droplet filling the space between the previously formed droplet and the new one. Eventually, the growing interface blocks the flow from the side channels, causing the upstream pressure in the continuous phase to increase until it reaches a critical value where the continuous phase begins to squeeze the interface and the necking stage begins (iii $\rightarrow$ iv). During the necking stage, the dispersed phase is still being injected into the droplet at a constant flow rate while the neck collapses. Once the neck reaches a critical size, the collapse accelerates, triggering the pinch-off stage (iv $\rightarrow$ v), which culminates with the droplet detaching. The newly formed droplet is pushed downstream and the cycle restarts. The pinch-off time is small and combined with the necking stage. The detailed definitions of filling stage and necking stage can be found in our previous study (Chen et al. 2015).

We use droplet volume, spacing and formation frequency as metrics to describe the output of the droplet generator. Droplet formation is considered as a quasi-steady state process where the flow rates are constant. Hence, the volume of each droplet is calculated as

$$
V_{d}=\frac{Q_{d}}{f}
$$

where $Q_{d}$ is the flow rate of the dispersed phase, and $f$ the frequency of droplet generation, which can be calculated by counting the number of droplets and frames captured during one cycle. The droplet volume could be experimentally measured and we compared the experimentally measured droplet volumes using the previously developed image analysis technique (T. Glawdel et al., 2012, Chen et al., 2015) with those obtained with Eqn (1) for several sets of experiments and noticed a small discrepancy of 
$\pm 5 \%$. Then we chose to use Eqn (1) for the droplet volume for this study due to its simplicity and the accuracy of the flow rate provided through our three in-line flow sensors.

$$
f=N_{\text {drop }} \frac{F}{N_{\text {frame }}}
$$

where $N_{\text {drop }}$ is the number of the captured droplets, $N_{\text {frame }}$ the total number of frames, and $F$ the frame rate.

With the same flow rates of both liquids and the same channel dimensions, Figure 2 shows that the filling time of $10 \%$ glyc $\left(\Delta t_{\text {filling_10\% }}=12 \mathrm{~ms}\right)$ is much longer than that of $80 \% \mathrm{glyc}$ $\left(\Delta t_{\text {filling_80\% }}=3.2 \mathrm{~ms}\right)$, which can be understood from the force balance. There are three forces acting on the droplet: shear force, interfacial tension force and pressure gradient. Since the droplet generation is confined in the squeezing regime ( $\mathrm{Ca}<0.006$ for all experimental conditions), the shear force can be ignored. Figure 2 shows the direction of interfacial tension force, which suspends the droplet from penetrating into the main channel. The interfacial tensions are similar for $10 \%$ glyc and $80 \%$ glyc which results in similar interfacial tension forces for both cases. However, the similar interfacial tension force cannot pull back $80 \% \mathrm{glyc}$ as much as it does for $10 \% \mathrm{glyc}$ due to the high viscosity of $80 \%$ glyc (i.e. $80 \%$ glyc has a higher viscosity, and higher hydrodynamic resistance, hence higher pressure under the same flow rates), as shown in stage (ii) in Figure 2 (b). As a result, $80 \%$ glyc quickly fills in the intersection causing the pressure in the continuous phase to build up and quickly reach the critical value due to the higher resistance associated with $80 \%$ glyc, which triggers the necking stage.

The necking time for $10 \%$ glyc $\left(\Delta t_{\text {necking_ } 10 \%}=5.8 \mathrm{~ms}\right)$ is shorter than that for $80 \%$ glyc $\left(\Delta t_{\text {necking_80\% }}=10.4 \mathrm{~ms}\right)$ because $80 \%$ glyc has a larger resistance to compression due to its high viscosity. The pinch-off position of $80 \% \mathrm{glyc}$ is lower than that of $10 \%$ glyc (see stage (iv) in Figure 2(b)). Although the necking time of $80 \% \mathrm{glyc}$ is longer than that of $10 \% \mathrm{glyc}$, its filling time is shorter. Therefore, the total droplet generation process for $80 \%$ gylc is shorter than that for $10 \%$ glyc resulting in a smaller droplet size which is scaled with the time of droplet formation. A comprehensive model for predicting the size of droplet generated in a flow focusing geometry with one single liquid as the dispersed phase has been previously reported for the squeezing regime (Chen et al. 2015)., expressed as,

$$
\frac{v_{d}}{w^{2} h}=\alpha+\beta \varphi
$$

where $V_{d}$ is the droplet volume, $w$ the channel width, $h$ the channel height, $\quad \alpha=\frac{Q_{d} \times \Delta t_{\text {filling }}}{w^{2} h}, \beta=\frac{Q_{c} \times \Delta t_{\text {necking }}}{w^{2} h}$, and $\varphi$ the flow rate ratio, $Q_{d} / Q_{C}$. Figure 2(c) shows that dimensionless droplet volume linearly increases with the flow rate ratio which agrees with Eq. (3). Empirical equations are obtained based on our experimental results with $V_{d}^{*}=1.31+1.41 \varphi$ for pure $10 \%$ glyc, and $V_{d}^{*}=0.83+1.55 \varphi$ for pure $80 \%$ glyc, respectively

\subsection{Droplet generation with stratified flow as the dispersed phase}

Droplet generation dynamics can be greatly influenced by the nonuniform viscosity of the dispersed phase. To study the influence of viscosity contrast on droplet generation, we considered a mixture of $10 \% \mathrm{glyc}$ and $80 \% \mathrm{glyc}$ as the dispersed phase where the flow rate ratio of $10 \%$ glyc to the total flow rate of the dispersed phase, $\lambda=Q_{10 \% \text { glyc }} /\left(Q_{10 \% \text { glyc }}+Q_{80 \% \text { glyc }}\right)$ was varied. For all the experiments the flow rate of the continuous phase and the total flow rate of the dispersed phase were fixed at $4 \mu \mathrm{l} / \mathrm{min}$ and $1.62 \mu \mathrm{l} / \mathrm{min}$, respectively, so that the flow rate ratio between the dispersed phase and the continuous phase, $\varphi$, was fixed at $\varphi=0.4$, which renders the flow in the squeezing regime. We varied $\lambda$ from $0 \sim 1$ where ' $\lambda=0$ ' represents pure $80 \% \mathrm{glyc}$ as the dispersed phase, while ' $\lambda=1$ ' represents pure $10 \% \mathrm{glyc}$ as the dispersed phase. The rest of experimental conditions are in the supplementary material, as Exp \#1 - \#7. Figure 3 shows the nondimensional droplet volumes with the variation of $\lambda$ for three cases.

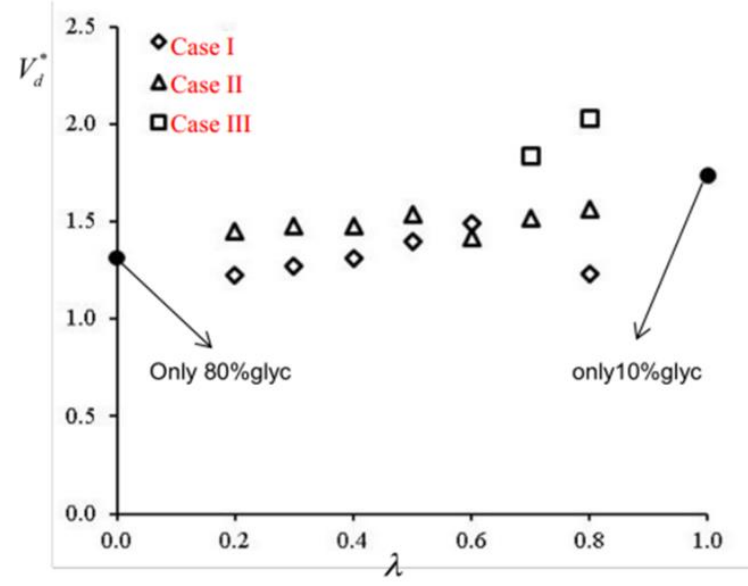

Figure 3. Non-dimensional droplet volume under different flow ratios of $10 \% \mathrm{glyc} /(10 \% \mathrm{glyc}+80 \% \mathrm{glyc})$, with a fixed flow rate of $Q_{\text {oil }}=$ $4 \mu \mathrm{l} / \mathrm{min}$ for the continuous phase and $Q_{10 \% \text { glyc }+80 \% \text { glyc }}=$ $1.62 \mu \mathrm{l} / \mathrm{min}$ for the dispersed phases. Non-dimensional droplet volume is defined as $V_{d}^{*}=V_{d} / w^{2} h$, where $w$ is the channel width, $h$ channel height. Case (I): low viscosity $10 \%$ glyc is surrounded by high viscosity $80 \%$ glyc; Case(II): $10 \%$ glyc and $80 \%$ glyc penetrate into the main channel side by side with $10 \%$ glyc coming from the top and $80 \%$ from the bottom; and Case(III): $80 \% \mathrm{glyc}$ is the inner fluid that is surrounded by $10 \%$ glyc.

Case I: In this case, low viscosity $10 \%$ glyc is surrounded by high viscosity $80 \%$ glyc. Droplet volume increases with the flow rate of $10 \%$ glyc when $\lambda<0.6$, however; decreases dramatically when $\lambda$ reaches 0.8 . At $\lambda=0.7$, droplets are generated with one bigger and one smaller droplet alternately. As discussed in section (A), the filling time depends on the resistance that the dispersed phase experiences when penetrating into the main channel, which is a combined effect of interfacial tension force for pulling back and pressure gradient for pinching during the formation cycle. Lower viscosity fluids have a longer filling stage and a shorter necking stage while higher viscosity fluids have a shorter filling stage and a longer necking stage. For stratified flow, the increase in pressure gradient in the continuous phase mainly depends on the fluid that contacts the continuous phase which is the outer layer of the fluid, so does the filling time. 


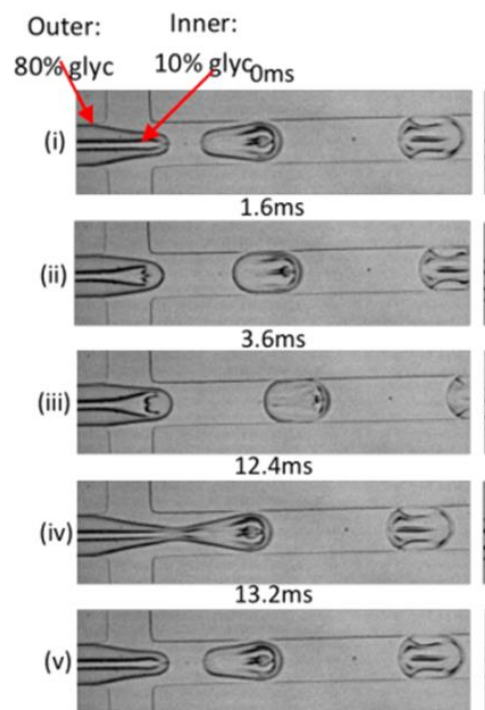

(a) $\lambda=0.2$

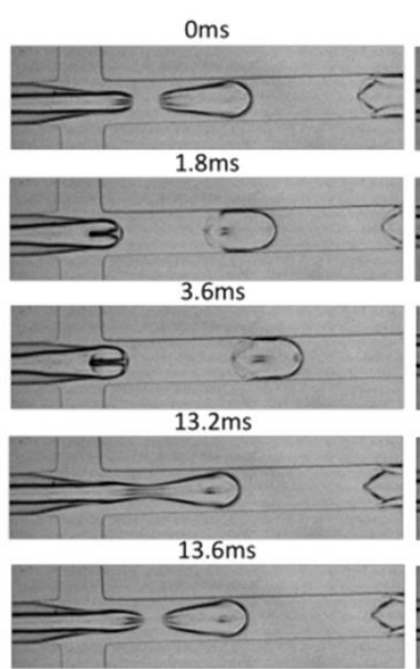

(b) $\lambda=0.7$

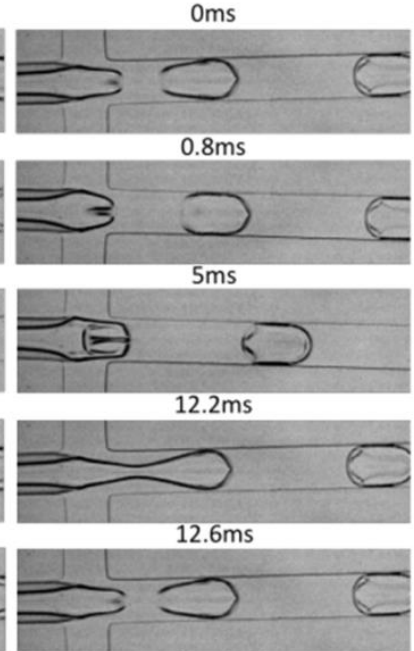

(c) $\lambda=0.8$

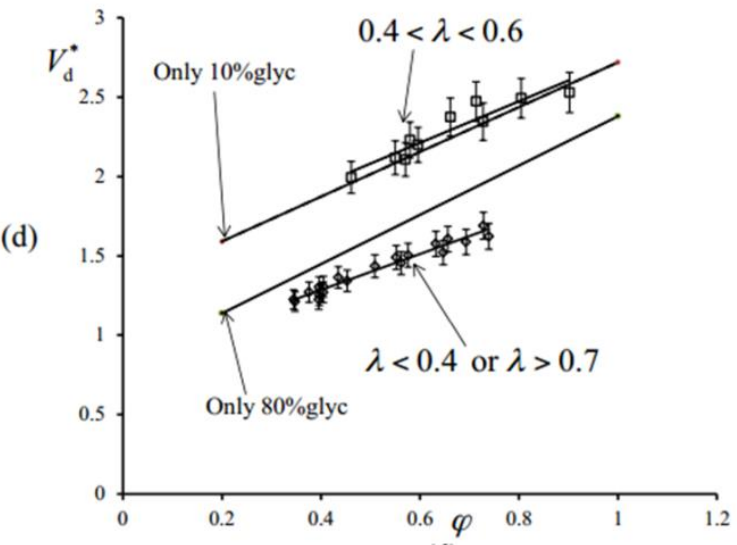

Figure 4. Droplet generation process: (i) Droplet pinches off; (ii) The dispersed phase returns back to the semilunar shape due to interfacial tension and starts to fill the main channel; (iii) End of filling stage where the silicone oil starts to squeeze the dispersed phase; (iv) The necking width reaches a critical value, and the droplet starts to pinch off; and (v) The current droplet pinches off, and a new droplet is forming. (a) $\lambda=$ $0.2, Q_{\text {oil }}=4 \mu \mathrm{l} / \mathrm{min}, Q_{10 \% g l y c}=0.32 \mu \mathrm{l} / \mathrm{min}, Q_{80 \% g l y c}=1.3 \mu \mathrm{l} / \mathrm{min} ;(\mathrm{b}) \lambda=0.7, Q_{\text {oil }}=4 \mu \mathrm{l} / \mathrm{min}, Q_{10 \% g l y c}=1.13 \mu \mathrm{l} / \mathrm{min}, Q_{80 \% g l y c}=$ $0.49 \mu \mathrm{l} / \mathrm{min} ;$ (c) $\lambda=0.8, Q_{\text {oil }}=4 \mu \mathrm{l} / \mathrm{min}, Q_{10 \% g l y c}=1.3 \mu \mathrm{l} / \mathrm{min}$ (d) The relationship between the measured droplet volume scaled as $V_{d}^{*}=$ $\frac{V_{d}}{w^{2} h}$ and flow rate ratio $\varphi=\left(Q_{10 \% g l y c}+Q_{80 \% g l y c}\right) / Q_{o i l}$, where the linear trend line for pure $10 \% \mathrm{glyc}$ and $80 \% \mathrm{glyc}$ can be predicted by $V_{d}^{*}=$ $1.31+1.41 \varphi$ and $V_{d}^{*}=0.83+1.55 \varphi$, respectively.

In Case I, when $\lambda$ is small, $80 \%$ glyc fully occupies the oilwater interface during the filling stage as shown in Figure 4 (a), and thus the filling time is close to that when $\lambda=0$ (the case of $80 \%$ glyc only) though the necking time is shorter because the inner fluid of $10 \%$ glyc reduces the resistance to compression during the necking process. Therefore, the resulting droplet size is smaller than that when $\lambda=0$. When $\lambda$ increases, the proportion of $10 \% \mathrm{glyc}$ in droplet will increase and will be partially present at the oil-water interface resulting in an increase in the filling time. Meanwhile, the necking time will decrease due to the increase of $10 \% \mathrm{glyc}$ in the neck region. Overall, the total time of the droplet generation process and droplet size will increase with $\lambda$.

When $\lambda$ increases to $0.7,10 \%$ glyc partially occupies the oilwater interface during the filling stage and necking stage, which creates two necks as shown in Figure 4(b). Therefore $\lambda=0.7$ will generate droplets with one bigger and one smaller alternately. It is a transition regime between small $\lambda$ and large $\lambda$. When $\lambda$ is large enough ( $\lambda=0.8$ in the experiment), $80 \%$ glyc is still on the oilwater interface during the filling stage, which leads to a short filling time, but $10 \%$ glyc fully occupies the neck during the necking stage, which leads to a very short necking time, as shown in Figure 4(c). Therefore, the droplet size will dramatically decrease, much smaller than when $\lambda=0$ (the only $80 \%$ glyc case). The dimensionless droplet volume is plotted in Figure 4 (d) as compared to pure $10 \%$ glyc case and pure $80 \%$ glyc case. When $\lambda<$ 0.4 or $\lambda>0.7$, droplet volume is smaller than only $80 \%$ glyc case, and roughly follows equation $V_{d}^{*}=0.83+1.13 \varphi$; when $0.4<$ $\lambda<0.6$, droplet size is close to that of only $10 \%$ glyc case, and roughly follows equation $V_{d}^{*}=1.43+1.31 \varphi$; when $0.6<\lambda<$ 0.7 , the flow starts generating one big and on small droplet alternatively.

Case II: in this case, $10 \%$ glyc and $80 \%$ glyc penetrate into the main channel side by side with $10 \%$ glyc coming from the top and $80 \%$ from the bottom. Each fluid occupies one side of the oil-water interface, both in the filling and necking stages, as shown in Figure $5(\mathrm{a} \sim \mathrm{c})$. Therefore, both the filling and necking times will be 


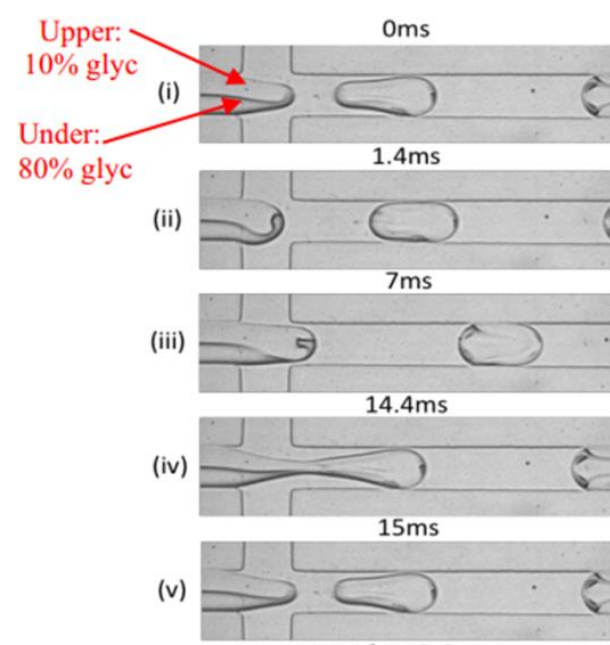

(a) $\lambda=0.2$
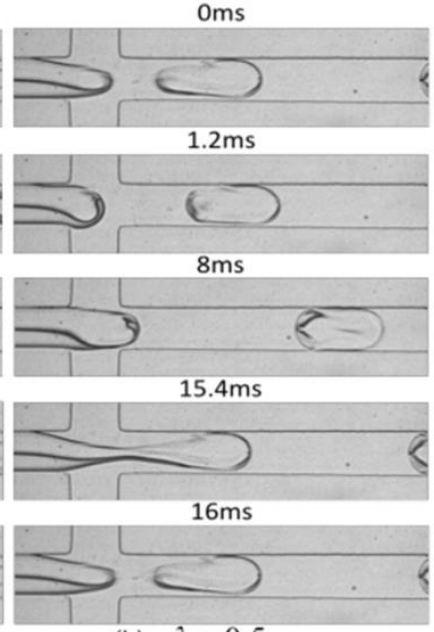

(b) $\lambda=0.5$
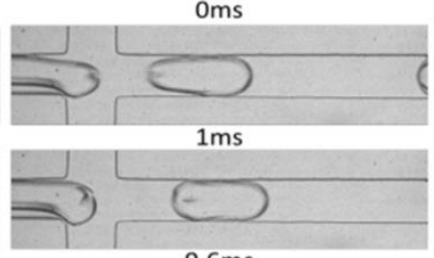

$9.6 \mathrm{~ms}$

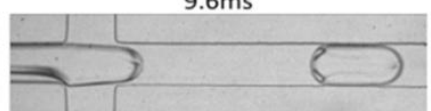

$15.8 \mathrm{~ms}$

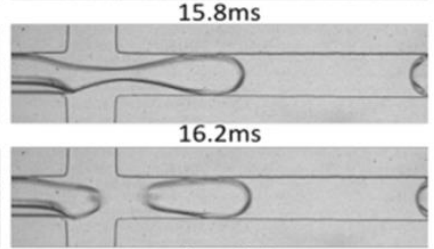

(c) $\lambda=0.8$

(d)

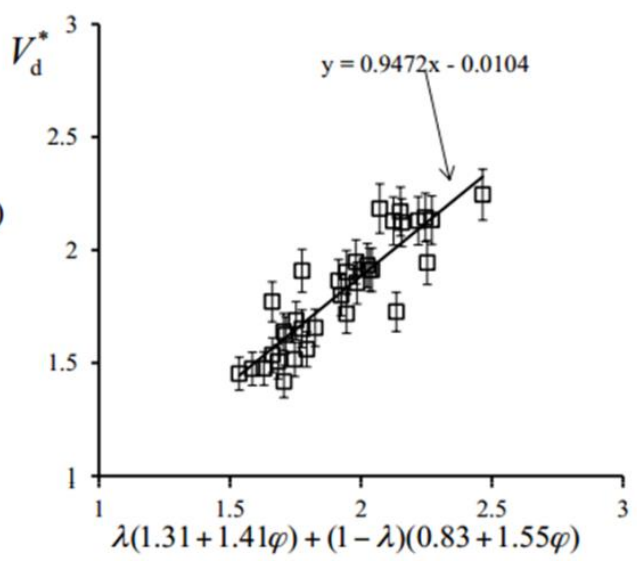

Figure 5. Droplet generation process (i) Droplet pinches off; (ii) The dispersed phase returns back to the semilunar shape due to interfacial tension and starts to fill the main channel; (iii) End of filling stage where the silicone oil starts to squeeze the dispersed phase; (iv) The necking width reaches a critical value, and the droplet starts to pinch off; (v) The current droplet pinches off, and a new droplet is forming. (a) $\lambda=$ $0.2, Q_{\text {oil }}=4 \mu \mathrm{l} / \mathrm{min}, \quad Q_{10 \% \text { glyc }}=0.32 \mu \mathrm{l} / \mathrm{min}, \quad Q_{80 \% \text { glyc }}=1.3 \mu \mathrm{l} / \mathrm{min} ; \quad$ (b) $\lambda=0.5, Q_{\text {oil }}=4 \mu \mathrm{l} / \mathrm{min}, Q_{10 \% g l y c}=0.81 \mu \mathrm{l} / \mathrm{min}$, $Q_{80 \% g l y c}=0.81 \mu \mathrm{l} / \mathrm{min} ;$ (c) $\lambda=0.8, Q_{o i l}=4 \mu \mathrm{l} / \mathrm{min}, Q_{10 \% g l y c}=1.30 \mu \mathrm{l} / \mathrm{min}, Q_{80 \% g l y c}=0.32 \mu \mathrm{l} / \mathrm{min}$; (d) The relationship between the measured droplet volume scaled as $V_{d}^{*}=V_{d} / w^{2} h$ and flow rate ratio.

between those for the cases with $\lambda=0$ and $\lambda=1$, and thus the droplet size will be between those two cases as well. The droplet size will increase with $\lambda$ because the droplet generation process will get close to the case with pure $10 \%$ glyc when $10 \%$ glyc proportion increases. Case II generates uniform droplets in the full range of $\lambda$. The droplet volume is between the case with pure $80 \%$ glyc and pure $10 \%$ glyc and can be estimated by a linear asymptotic model:

$$
V_{\dot{d}}^{*}=\lambda(1.31+1.41 \varphi)+(1-\lambda)(0.83+1.55 \varphi)
$$

One can see that when $\lambda=0$, it approaches to the model of pure $80 \% \mathrm{glyc}$, and when $\lambda=1$, it approaches to the model of only $10 \%$ glyc. Figure $5(\mathrm{~d})$ shows that this estimation agrees well with our experimental results.

Case III: in this case, $80 \% \mathrm{glyc}$ is the inner fluid that is surrounded by $10 \%$ glyc. When $\lambda \leq 0.6,80 \%$ glyc will occupy most of the channel cross-section due to its low velocity. The $80 \%$ glyc plays a role like a 'soft wall'. This case will create two necks during the necking stage. Therefore, it generates two different sizes of droplets alternately, as shown in Figure 6(a). When $\lambda \geq 0.7$, it starts generating uniform droplets as shown in Figure 6(b). The $10 \%$ glyc fully occupies the oil-water interface during the necking stage. Hence, the filling time is almost the same as for $\lambda=1$ (the only $10 \%$ case). During the necking stage, the neck consists of both $10 \% \mathrm{glyc}$ and $80 \% \mathrm{glyc}$, which increases the resistance to compression compared to the case with $\lambda=1$. Therefore, the necking time is longer than when $\lambda=1$, leading to droplet size larger than 'pure $10 \%$ glyc case'. The equation for calculating droplet size in case (III) with $\lambda \geq 0.7$ can be estimated as,

$$
V_{d}^{*}=1.31+\frac{1.41}{\lambda^{m}} \varphi
$$

where the constant $\alpha=1.31$ is set to be the same as pure $10 \% \mathrm{glyc}$ case, since the filling time is almost the same as for $\lambda=01$. $\beta$ is a power function of $\lambda$, and when $\lambda=1$, Eqn (5) simplifies to the model for pure $10 \%$ glyc case. Figure 6 (c) shows that droplet volume in case (III) is the largest compared to other cases. We 


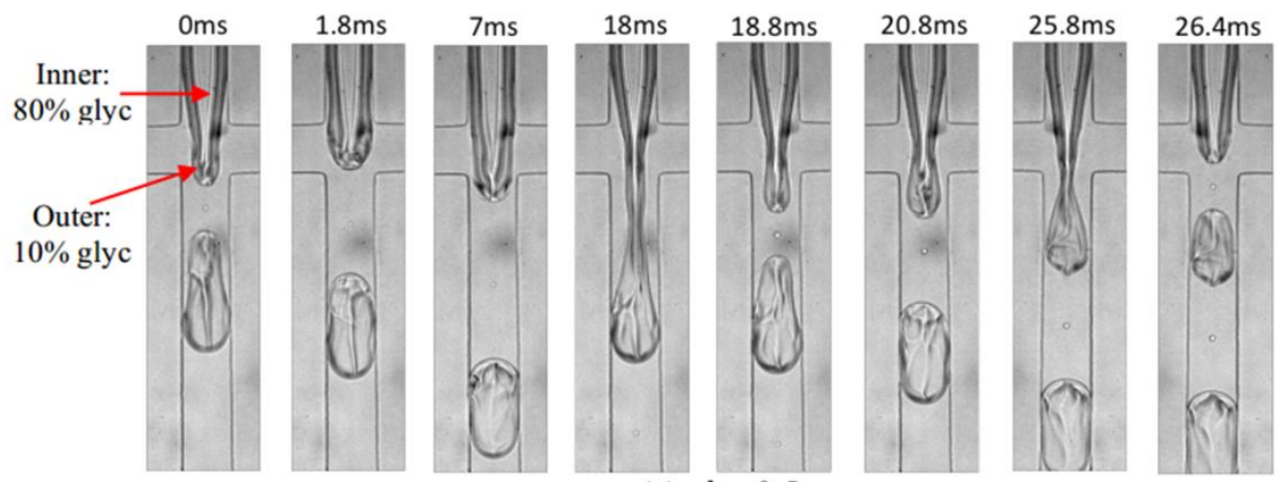

(a) $\lambda=0.5$

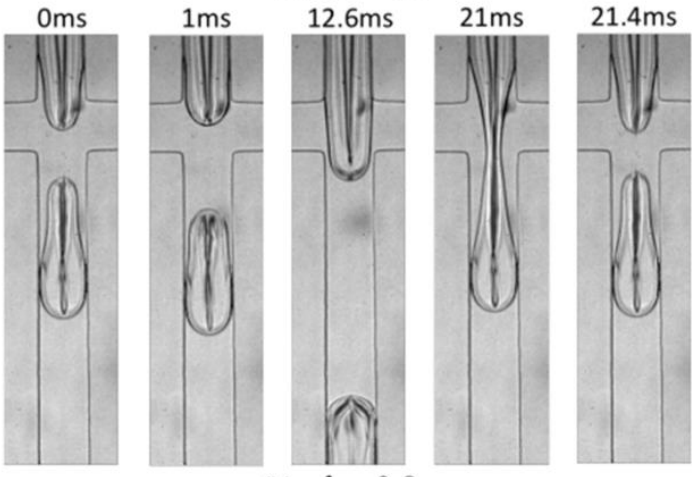

(b) $\lambda=0.8$
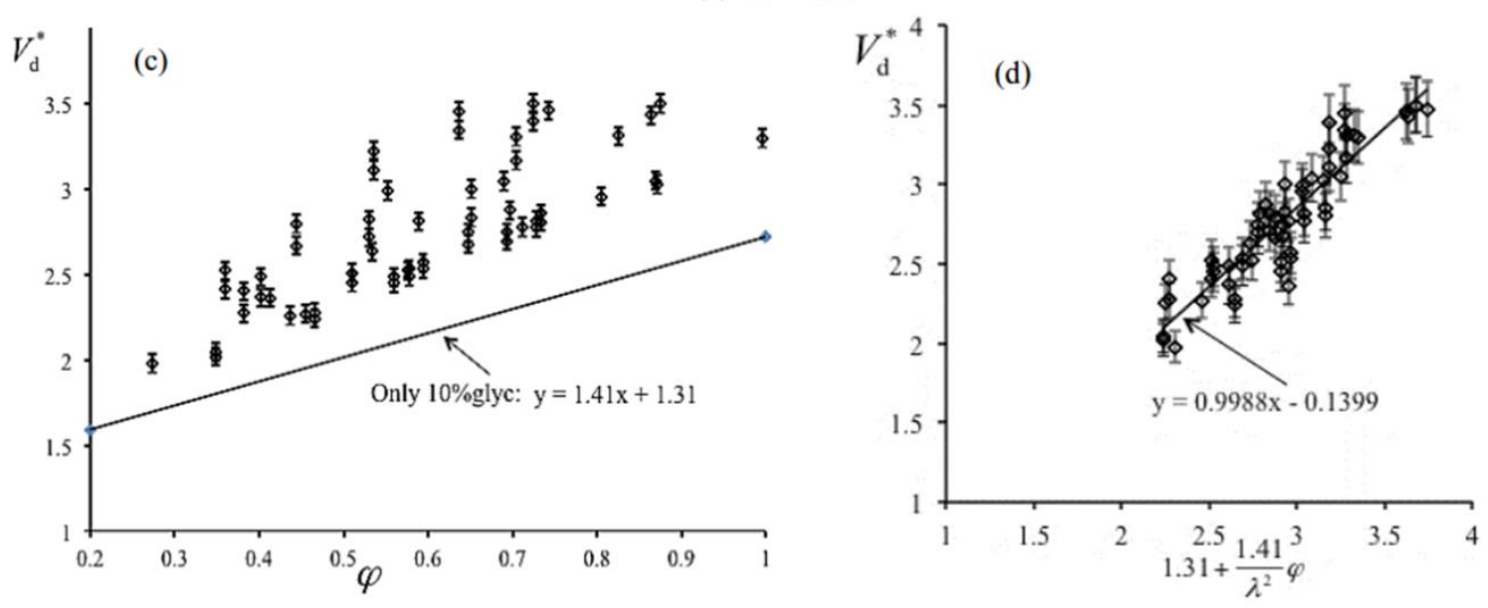

Figure 6. Droplet generation process with (a) $\lambda=0.5, Q_{\text {oil }}=4 \mu \mathrm{l} / \mathrm{min}, Q_{10 \% g l y c}=0.81 \mu \mathrm{l} / \mathrm{min}, Q_{80 \% g l y c}=0.81 \mu \mathrm{l} / \mathrm{min}$; (b) $\lambda=0.8, Q_{\text {oil }}=$ $4 \mu \mathrm{l} / \mathrm{min}, Q_{10 \% g l y c}=1.30 \mu \mathrm{l} / \mathrm{min}, Q_{80 \% g l y c}=0.32 \mu \mathrm{l} / \mathrm{min}$; (c) and (d) The relationship between the measured droplet volume scaled as $V_{d}^{*}=$ $\frac{V_{d}}{w^{2} h}$ with the flow rate ratio, $\varphi$, and the formula of $1.31+\frac{1.41}{\lambda^{2}} \varphi$, respectively.

figured out that when $m=2.1$, this model fits experimental results very well as shown in Figure 6(d). From Figure 6(d), we can see that there exists a bias error of 0.14 , which is because the filling time of case (III) should be a little bit smaller than that of pure $10 \%$ glyc case due to the presence of $80 \%$ glyc in case (III). Therefore, when $\lambda \geq 0.7$, the droplet volume of case (III) can be estimated as,

$$
V_{d}^{*}=1.31+\frac{1.41}{\lambda^{2.1}} \varphi-0.14=1.17+\frac{1.41}{\lambda^{2.1}} \varphi
$$

3.3. Stratified flow with a viscosity contrast and its impact on single particle encapsulation
We studied the stratified flow with viscosity contrast under three cases: Case I - low viscosity fluid surrounded by high viscosity fluid; Case II -high viscosity fluid parallel to low viscosity fluid; Case III -high viscosity fluid surrounded by low viscosity fluid, as shown in Figure 7.

The stratified flow with a viscosity contrast in a rectangular channel can be considered as the Poiseuille's flow due to its low Reynolds number. Hence, the velocity profile can be considered as a parabola. When the two different fluids go into the first junction, there exists a velocity contrast at the interface, as illustrated in Figure 7(b). Therefore, a shear force is present at the interface when the two fluids meet, which tends to increase the velocity of 
$80 \%$ glyc and reduce the velocity of $10 \%$ glyc until there is no velocity contrast at the interface. The stream width of each flow can be expressed as:

$$
\varepsilon \propto \frac{Q}{\bar{v}}
$$

where $\varepsilon$ is the stream width, $Q$ the flow rate, and $\bar{v}$ the areaaverage velocity. When the viscous stratified flow moves downstream, $\bar{v}_{80 \% g l y c}$ will increase and $\bar{v}_{10 \% g l y c}$ will decrease due to the shear force on the interface. Hence, $\varepsilon_{10 \% \text { glyc }}$ will increase and $\varepsilon_{80 \% g l y c}$ will decrease. These findings were verified by experimental results, as shown in Figure 7(a), where one can see that $80 \%$ glyc speeds up and becomes thinner as it moves along the channel. When the stratified flow penetrates into the silicone oil, $80 \%$ glyc keeps speeding up and occupies the front tip, then is forced back due to the interfacial tension.

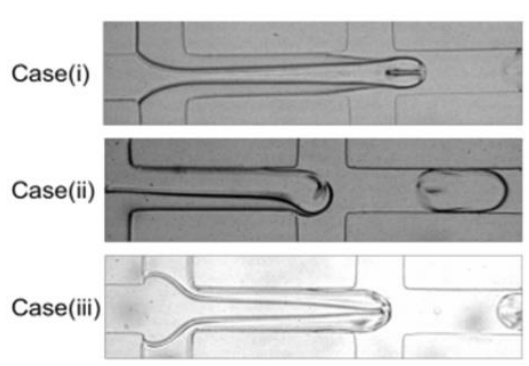

(a)

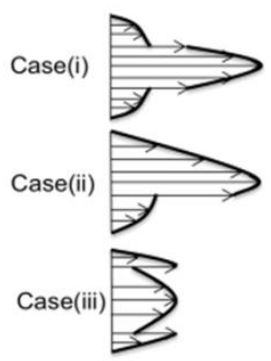

(b)
Figure 7. (a) Viscosity-stratified flow shapes when the dispersed phases start filling the main channel with flow rates $Q_{\text {oil }}=$ $4 \mu \mathrm{l} / \mathrm{min}, Q_{10 \% g l y c}=1.13 \mu \mathrm{l} / \mathrm{min}, Q_{80} \%$ glyc $=0.49 \mu \mathrm{l} / \mathrm{min}$. Case I: $10 \%$ glyc is surrounded by $80 \%$ glyc, Case II: $10 \%$ glyc is parallel to $80 \%$ glyc, and Case III: $80 \%$ glyc is surrounded by $10 \%$ glyc. (b) Sketch of the velocity profile of viscous stratified flows in rectangular channel at the first junction for three cases.

Figure 7 (a) indicates that Case III is the best choice for encapsulating single (bio)particles within droplets compared to the other two cases, because the inner stream (i.e. high viscosity $80 \% \mathrm{glyc}$ ) is being gradually focused downstream. If (bio)particles are suspended in this fluid, they can be arranged in line and separated to ensure droplets being spaced out. Each method has pros and cons. Despite the success of the existing methods for single (bio)particle encapsulation, it is clear that this method offers a few advantages. For example, it avoids the blockage problem that is often seen in the designs with high aspect-ratio channels as the high viscosity fluid acts as 'soft wall' and the width of that 'soft wall' can be controlled relatively easily by varying the aqueous flow rates. In addition, high aspect ratio channels are challenging to achieve with PDMS material due to its softness. In cases I and II, the middle stream where (bio)particles are supposed to be suspended cannot be focused and thus are difficult to be aligned and synchronized with droplet generation.

To demonstrate the effect of the flow focusing structure observed in Case III on single (bio)particle encapsulation, polystyrene microbeads (Polysciences, Inc.) with a diameter of 10 um were added to the $80 \%$ glyc solution. The particle suspension had a very high concentration of $1 \times 10^{7}$ particles $/ \mathrm{ml}$ after being mixed with $80 \%$ glyc. The particle ordering and subsequent encapsulation are shown in Figure 8 (Multimedia view). Single (bio)particle encapsulation was greatly improved with a stratified flow of Case III where beads are focused and spaced (Figure 8(b)) and then encapsulated in droplets. On average, the single particle encapsulation is achieved with an efficiency of around $52 \%$ (Multimedia view) although the image shows in Figure 8 a bit higher $(\sim 80 \%)$. Whereas for the case without stratified flow, encapsulation of single or more particles was observed.

It is noticed that this method is not as good as the reported methods which achieved as high as $98 \%$ single encapsulation. This is largely attributed to some practical issues such as particle aggregation and forced particle dilution. The focusing of particle is achieved by the use of high viscous sample fluid helping particles stay within the sample fluid and the drag force at the interface between the slow sample fluid and the fast moving focusing fluids which 'thins' the sample fluid. The spacing of droplets is mainly achieved by the fast moving focusing fluid which prevents multiple particles to enter the first junction the same time. The whole strategy works well if the particle concentration is high resulting packed particles waiting at the first junction to be released and spaced in between. However, when the particle concentration is high, aggregation occurs which limits the concentration that can be used practically. Another practical challenge is the operation is prone to dust at the inlets and in the channel, which slow down the particle and thus dilutes the particle concentration downstream.

\section{Conclusion}

This work experimentally studied the droplet generation process in a flow focusing device with two junctions in series where two miscible fluids with a viscosity contrast are used as the dispersed phase. By systematically investigating the droplet size and formation frequency for five scenarios and developing models for droplet size for each scenario, it is found that viscosity contrast in the dispersed fluids has significant impact on droplet formation dynamics and thus presents unique opportunity as a controlling parameter for tailoring generator design towards specific applications. In particular, case III which has high viscous $80 \%$ glyc surrounded by $10 \%$ glyc in the first junction generates the largest droplet size, even larger than that in Ref Case I with pure $10 \%$ glyc case as the dispersed phase. In addition, the focusing structure has proven useful for helping the ordering of particles and thus has potential to improve single encapsulation performance. Droplet sizes in case II are between the cases with pure $10 \%$ glyc (Ref Case I) and pure $80 \%$ glyc (Ref Case II). Case II generates stable and monodispersed droplets in a larger range of flow ratios between each reagent, which is useful for high throughput reactions requiring uniform droplet size. Droplet sizes in case I which has $10 \%$ glyc surrounded by $80 \%$ glyc in the first junction depend on the flow percentage of $10 \% \mathrm{glyc}$ and will fall into different regimes.

\section{Acknowledgments}

The authors gratefully acknowledge the support from Canada Natural Science and Engineering Council of Canada, Canada Foundation for Innovation, Canada Research Chair program, and Advanced Electrophoresis Solutions Ltd, through grants to Dr. Carolyn L. Ren. 


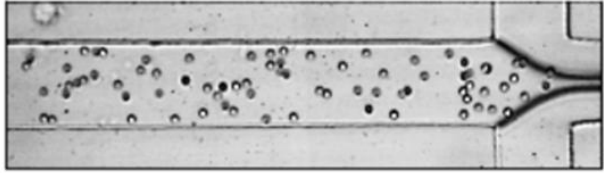

(a) Microbeads at upstream

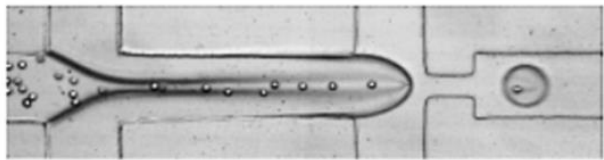

(b) Mierobeads ordering

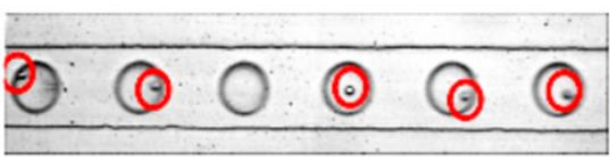

(c) Microbeads encapsulated in droplets

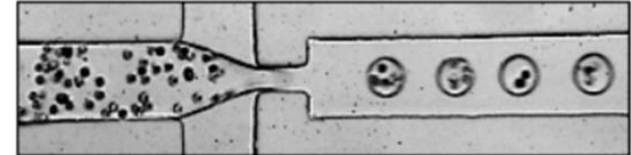

(d) Droplets generation without stratified flow

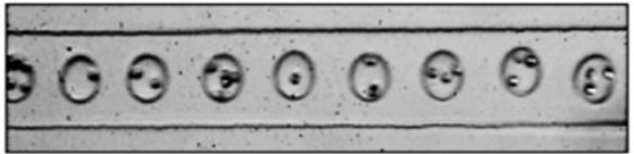

(e) Microbeads encapsulated in droplets without stratified flow

Figure 8. Images captured from experimental videos showing single particle encapsulation with a stratified flow of case (III) (a $\sim$ ) (MULTIMEDIA VIEW) and without a stratified flow (d e)

\section{References}

Abate, A. R., Chen, C., Agresti, J., Weitz, D. A., 2009. Beating Poisson encapsulation statistics using close-packed ordering. Lab Chip 9, 2628-2631.

Bai, L., Fu, Y., Zhao, S., Cheng, Y., 2016. Droplet formation in a microfluidic T-junction involving highly viscous fluid systems. Chem. Eng. Sci.145,141-148.

Berthier, J., Vot, S. L., Tiquet, P., David, N., Lauro, D., Benhamou, P. Y., Rivera, F., 2010. Highly viscous fluids in pressure actuated flow focusing devices. Sensors Actuators A 158, 140-148

Casadevall i Solvas, X., DeMello, A., 2011. Droplet microfluidics: recent developments and future applications. Chem. Commun. (Camb) 47, 1936-1942.

Chen, X., Glawdel, T., Cui, N., Ren, C. L., 2015. Model of droplet generation in flow focusing generators operating in the squeezing regime. Microfluid. Nanofluid. 18, 1341-1353.

Cubaud, T., Jose, B. M., Darvishi, S., Sun, R., 2012. Droplet breakup and viscosity-stratified flows in microchannels. Int. J. of Multiphase Flow 39, 29-36.

Cubaud, T., Mason, T. G., 2008. Capillary threads and viscous droplets in square microchannels. Phys. Fluids 20, 053302.

Edd, J. F., Di Carlo, D., Humphry, K. J., Köster, S., Irimia, D., Weitz, D. A., Toner, M., 2008. Controlled encapsulation of single-cells into monodisperse picolitre drops. Lab Chip 8, 1262-1264.

Fu, T., Ma, Y., Funfschilling, D., Li, H., 2009. Bubble formation and breakup mechanism in a microfluidic flow-focusing device. Chem. Eng. Sci. 64, 2392-2400.

Fu, T., Wu, Y., Ma, Y., Li, H., 2012. Droplet formation and breakup dynamics in microfluidic flow-focusing devices: From dripping to jetting. Chem. Eng. Sci. 84, 207-217.

Garstecki, P., Fuerstman, M. J., Stone, H. A., Whitesides, G. M., 2006. Formation of droplets and bubbles in a microfluidic T-junctionscaling and mechanism of break-up. Lab Chip 6, 437-446.

Glawdel, T., Elbuken, C., Ren, C. L., 2012. Droplet formation in microfluidic T-junction generators operating in the transitional regime. I. Experimental observations. Phys. Rev. E 85, 016322.

Glawdel, T., Elbuken, C., Ren, C. L, 2011. Passive droplet trafficking at microfluidic junctions under geometric and flow asymmetries. Lab Chip 11, 3774-3784.

Haeberle, S., Zengerle, R., 2007. Microfluidic platforms for lab-on-achip applications. Lab Chip 7, 1094-1110.
Jouyban, A., 2008. Review of the cosolvency models for predicting solubility of drugs in water-cosolvent mixtures. J. Pharm. Pharmaceut. Sci. 11, 32-58.

Kemna, E.W.M., Schoeman, R. M., Wolbers, F., Vermes, I., Weitz, D. A., van den Berg, A., 2012. High-yield cell ordering and deterministic cell-in-droplet encapsulation using Dean flow in a curved microchannel. Lab Chip 12, 2881-2887.

Kim, Y. H., Zhang, L., Yu, T., Jin, M., Qin, D., Xia, Y., 2013. DropletBased Microreactors for Continuous Production of Palladium Nanocrystals with Controlled Sizes and Shapes. Small 9, 34623467.

Korczyk, P. M., Cybulski, O., Makulska, S., Garstecki, P., 2011. Effects of unsteadiness of the rates of flow on the dynamics of formation of droplets in microfluidic systems. Lab Chip 11, 173175.

Köster, S., Angilè, F. E., Duan, H., Agresti, J. J., Wintner, A., Schmitz, C., Rowat, A. C., Merten, C. A., Pisignano, D., Griffiths, A. D, Weitz, D. A., 2008. Drop-based microfluidic devices for encapsulation of single cells. Lab Chip 8,1110-1115.

Kwapiszewska, K., Michalczuk, A., Rybka, M., Kwapiszewski, R., Brzózka, Z., 2014. A microfluidic-based platform for tumour spheroid culture, monitoring and drug screening. Lab Chip 14, 2096-2104.

Lagus, T. P., Edd, J. F., 2013. A review of the theory, methods and recent applications of high-throughput single-cell droplet microfluidics. J. Phys. D: Appl. Phys. 46, 114005.

Lee, W., Walker, L. M., Anna, S. L., 2009. Role of geometry and fluid properties in droplet and thread formation processes in planar flow focusing. Phys. Fluids 21, 032103.

Lu, Y., Fu, T., Zhu, C., Ma, Y., Li, H., 2014. Scaling of the bubble formation in a flow-focusing device: Role of the liquid viscosity. Chem. Eng. Sci. 105, 213-219.

Miller, O. J., El, A., Mangeat, T., Baret, J., Frenz, L., El, B., Mayot, E., Samuels, M. L., Rooney, E. K., Dieu, P., Galvan, D. R., Link, M., Griffiths, A. D., 2012. High-resolution dose-response screening using droplet-based microfluidics. Proc. Natl. Acad. Sci. U.S.A. 109, 378-383.

Rosenfeld, L., Lin, T., Derda, R., Tang, S. K. Y., 2014. Review and analysis of performance metrics of droplet microfluidics systems. Microfluid. Nanofluidics 16,921-939.

Rossow, T., Heyman, J. A., Ehrlicher, A. J., Langhoff, A., Weitz, D. A., Haag, R., Seiffert, S., 2012. Controlled synthesis of cell-laden 
microgels by radical-free gelation in droplet microfluidics. J. Am. Chem. Soc. 134, 4983-4989.

Schaerli, Y., Wootton, R. C., Robinson, T., Stein, V., Dunsby, C., Neil, M., French, P. M. W., Demello, A. J., Abell, C., Hollfelder, F., 2009. Continuous-flow polymerase chain reaction of singlecopy DNA in microfluidic microdroplets. Anal. Chem. 81, 302306.

Shepherd, R.F., Conrad, J. C., Rhodes, S. K., Link, D. R., Marquez, M., Weitz, D. A., Lewis, J. A., Program, P., Di, V., Road, C., 2006. Microfluidic Assembly of Homogeneous and Janus Colloid-Filled Hydrogel Granules. Langmuir 18, 8618-8622.

Shestopalov, I., Tice, J. D., Ismagilov, R. F., 2004. Multi-step synthesis of nanoparticles performed on millisecond time scale in a microfluidic droplet-based system. Lab Chip 4, 316-321.

van Steijn, V., Kleijn, C. R., Kreutzer, M. T., 2010. Predictive model for the size of bubbles and droplets created in microfluidic Tjunctions. Lab Chip 10, 2513-2518.
Tewhey, R., Warner, J. B., Nakano, M., Libby, B., Medkova, M., David, P. H., Kotsopoulos, S. K., Samuels, M. L., Hutchison, J. B., Larson, J. W., Topol, E. J., Weiner, M. P., Harismendy, O., Olson, J., Link, D. R., Frazer, K. A., 2009. Microdroplet-based PCR enrichment for large-scale targeted sequencing. Nat. Biotechnol. 27, 1025-1031.

Velasco, D., Tumarkin, E., Kumacheva, E., 2012. Microfluidic encapsulation of cells in polymer microgels. Small 8, 1633-1642.

Wacker, J. B., Lignos, I., Parashar, V. K., Gijs, M. A. M., 2012. Controlled synthesis of fluorescent silica nanoparticles inside microfluidic droplets. Lab Chip 12, 3111-3116.

Wu, L., Chen, P., Dong, Y., Feng, X., Liu, B. F., 2013. Encapsulation of single cells on a microfluidic device integrating droplet generation with fluorescence-activated droplet sorting. Biomed. Microdevices 15, 553-560. 